\title{
A Busca de fármacos para tratamento da Tripanossomíase Americana: 103 anos de negligência
}

\author{
Whatani Silva Bezerra*, Dionatas Ulises de Oliveira Meneguetti**, \\ Luis Marcelo Aranha Camargo***
}

RESUMO: A presente revisão objetivou descrever um histórico da busca de fármacos para tratamento da Tripanossomíase Americana, e sua situação atual. A pesquisa foi desenvolvida através de revisão bibliográfica do tipo exploratória, retrospectiva e descritiva baseada em livros e pesquisa de artigos em base de dados Scientific Electronic Library Online (Scielo), Biblioteca Virtual em Saúde (BVS) e National Institute of Health (PUBMED), utilizando os descritores: Tripanossomíase Americana, tratamento e perspectivas. Já se passaram mais de 100 anos da descoberta da doença de Chagas, e até hoje não foi desenvolvido nenhum fármaco com potencial satisfatório de cura. 0 medicamento ainda utilizado é o benzonidazol, utilizado em combinação com outros medicamentos. Entre os quimioterápicos o Posaconazol, é o mais forte candidato para novos tratamentos específicos da doença e, entre os fitoterápicos, citam-se as espécies Banisteriopsis caapi e Psychotria viridis, utilizadas na região amazônica como alternativa.

Descritores: Tripanossomíase Americana, Tratamento, Perspectivas.

\section{The pursuit of drugs for Chagas disease treatment (American trypanosomiasis): 103 years of neglect}

\begin{abstract}
This review aimed to describe a historical pursuit of drugs for American trypanosomiasis treatment, and its current status. The survey was developed through exploratory and descriptive literature review based on books and articles in Scientific Electronic Library Online (SciELO), Virtual Health Library (VHL), and National Institute of Health (PubMed) databases using the keywords: American trypanosomiasis, treatment and perspectives. It has been more than 100 years since the discovery of Chagas disease, and no drug has been developed with suitable potential to cure it. Benznidazole in association with other drugs is still used. Among the chemotherapeutic drugs, Posaconazole is the strongest candidate for new disease-specific treatments. Among the herbal medicines Banisteriopsis caapi and Psychotria viridis are cited, used in the Amazon region as an alternative.
\end{abstract}

Descriptors: American trypanosomiasis, treatment and perspectives.

\footnotetext{
*Aluno do Curso de Graduação em Farmácia Generalista da Faculdade de Educação e Meio Ambiente (FAEMA), Ariquemes, RO, Brasil.

**Mestre em Genética e Toxicologia Aplicada, Docente e Coordenador de Extensão da Faculdade de Educação e Meio Ambiente (FAEMA), Ariquemes, RO, Brasil.

***Doutor em Ciências (Biologia da Relação Patógeno-Hospedeiro), Docente e Coordenador do Curso de Medicina da Faculdade São Lucas (FSL), Responsável pelo Instituto de Ciências Biomédicas ICB-5-USP, Monte Negro, RO, Brasil.
} 
Introdução

A Tripanossomíase Americana conhecida popurlamente com doença de Chagas (DC), foi descoberta em 1908 pelo médico brasileiro Carlos Chagas e, coube ao mesmo, diagnosticar e estudar clinicamente o primeiro caso humano da tripanossomíase em uma criança no ano de $1909 .{ }^{1,2}$ A DC é o único exemplo da história em que o agente causal foi descoberto antes da doença propriamente dita. ${ }^{3}$ Com a descoberta, Carlos Chagas homenagiou o epidemiologista Oswaldo Cruz com o seu nome no agente causador Trypanosoma cruzi., 4

Considerada endêmica em países da América Latina e Central, a doença vem se disseminando também em outras partes do mundo como resultado da imigração. São cada vez mais frequentes os registros do mal nos Estados Unidos, em países da Europa, na Austrália e no Japão. ${ }^{6}$

Em todo o mundo, mais de 14 milhões de pessoas estão infectados pelo parasita causador da DC, ${ }^{4}$ sendo estimado que cerca de 100 milhões de pessoas ainda correm 0 risco de contrair esta doença. ${ }^{8} \mathrm{~A}$ incidência anual é de 200 mil novos casos registrados em quinze países. ${ }^{9}$

A DC é um problema atual, global e negligenciado, ela não interessa à indústria farmacêutica pelo perfil econômico dos pacientes, serem geralmente, de pessoas economicamente desfavorecidas. A doença também não tem sido assistida de maneira apropriada pelos governos e ainda é pouco conhecida até pelos médicos, cuja formação conta com pouquíssimas horas sobre o assunto em algumas instituições, diz o médico David de Souza, coordenador da Unidade Médica de MSF Brasil. ${ }^{6}$

A DC está enquadrada em um grupo de enfermidades onde são conhecidas como "Doenças Negligenciadas", que recebem esse nome por se tratarem de doenças que acometem milhares de pessoas em sua grande maioria com baixa renda e em países subdesenvolvidos. Acredita-se que o pouco investimento no tratamento e profilaxia das mesmas, é devido ao nível de população onde normalmente elas ocorrem. ${ }^{10}$

Entre as doenças que pertencem a esse grupo, podemos citar além da DC, a malária, leishmanioses, filarioses e a dengue. Dados divulgados pelo órgão internacional, Drugs for Neglected Diseases Initiative ${ }^{10}$, relatam que apenas 1\% dos 1.393 novos fármacos registrados entre 1975 e 1999 foram destinados às doenças tropicais. Esses números revelam a existência de uma política de pesquisa excludente, na qual apenas $10 \%$ da despesa mundial com pesquisa em saúde são gastos em doenças que representam $90 \%$ da carga global. Pesquisadores de todo o mundo, principalmente os latino-americanos, têm trabalhado para fornecer à população necessitada, alternativas para este tratamento que, na maioria dos casos, se apresenta de forma inadequada. ${ }^{11}$

$\mathrm{Na}$ América Latina, essa doença figura entre as quatro principais endemias, sendo um dos seus maiores problemas sanitários, afetando principalmente as populações dos países pobres do continente americano., ${ }^{3,12,13}$ Os países endêmicos compreendem países da América do Sul, como Colômbia, Venezuela, Equador, Peru, Brasil, Bolívia, Chile, Uruguai, Argentina, e da América Central o México.,13

Saúde (Santa Maria), v.38, n.1, p. 9-20, 2012. A busca de fármacos para tratamento da Tripanossomiase Americana: 103 anos de negligência ISSN 2236-5834 
Até o início de 1990, a DC foi classificada pelo Banco Mundial como a mais séria das doenças parasitárias na América Latina, com um impacto socioeconômico (medido como DALY - Disability-Adjusted Life Years) consideravelmente maior do que os efeitos combinados de todos os outras infecções parasitárias. ${ }^{14,15}$

No Brasil, a estimativa é que existam cinco milhões de pessoas infectadas, com maior prevalência nos estados do Nordeste..$^{16,17}$ No Brasil, a DC foi responsável por cerca de 16 mil mortes por ano nas duas últimas décadas e entre 10.000 a 20.000 novos casos surgem anualmente. $3,18,19$

O presente estudo objetivou descrever um histórico da busca de fármacos para tratamento da Tripanossomíase Americana, e sua situação atual.

\section{Metodologia}

A presente pesquisa foi desenvolvida através de revisão bibliográfica do tipo exploratória descritiva, baseada em pesquisa de livros da biblioteca Julio Bordignon, pertencente à Faculdade de Educação e Meio Ambiente (FAEMA).

Também foram pesquisados artigos em base de dados Scientific Electronic Library Online (Scielo), Biblioteca Virtual em Saúde (BVS) e National Institute of Health PUBMED, utilizando os descritores: Tripanossomíase Americana, tratamento e perspectivas, sendo realizado 0 cruzamento entre as mesmas. Foram pesquisados artigos nas línguas portuguesa, inglesa e espanhol, contendo textos compreendidos entre o período de 1916 a 2010, e outros quando necessário devido sua grande relevância para a pesquisa. A utilização de uma quantidade elevada de trabalhos publicados a mais de 10 anos, se deve a importância dos mesmos, por descreverem as tentativas de se obter fármacos eficientes para o tratamento da Tripanossomíase Americana.

\section{Sintomas}

A doença se caracteriza pela sua variedade nas apresentações clínicas. Em geral, apresenta uma fase aguda (aparente e Inaparente) e uma fase crônica (indeterminada assintomática, ou sintomática tardia cardíaca e/ou digestiva). ${ }^{20,21,22}$

A fase aguda ou inicial da doença, quando aparente, é caracterizada por uma miocardite, na maioria das vezes, só traduzível eletrocardiograficamente. As manifestações gerais são de febre, mal-estar, cefaléia, astenia, hiporexia, hipertrofia de linfonodos, hepatoesplenomegalia e com pouca frequência meningoencefalite. Os sinais mais característicos da fase aguda são: o chagoma que é um inchaço na região da picada ${ }^{12}$ e 0 sinal de Romana que é um edema bipalpebral quando a transmissão ocorre pelo olho, ${ }^{23}$ as duas aparecem de sete a dez dias após a infecção e permanecem por cerca de dois a quatro meses. $^{24,25}$

A fase crônica é classificada sob três formas clínicas: indeterminada, cardíaca e digestiva. ${ }^{25} \mathrm{Na}$ forma indeterminada, as alterações patológicas são pouco significativas, o que torna o diagnóstico clínico difícil; nesta fase, os testes sorológicos geralmente são positivos, ${ }^{26}$ as pesquisas apontam que cerca de $60 \%$ das pessoas infectadas se encontram nesta forma. ${ }^{25} \mathrm{~A}$ forma cardíaca é a mais importante, se caracteriza por uma limitação ao doente

Saúde (Santa Maria), v.38, n.1, p. 9-20, 2012. Bezerra, W. S.; Meneguetti, D. U. O.; Camargo, L. M. A. ISSN 2236-5843 
chagásico e a principal causa de morte, ${ }^{27,28}$ Apresenta-se, geralmente, sem sintomatologias com alterações eletrocardiográficas, quando ocorrem, os sinais e sintomas apresentados são: palpitações, dispnéia, edema, dor precordial e tonturas, $, 29,30,31$ podendo levar à síndrome de insuficiência cardíaca progressiva, insuficiência cardíaca fulminante ou arritmias graves e morte súbita. A forma digestiva caracteriza-se por alterações ao longo do trato digestivo, ocasionadas por lesões dos plexos nervosos, com consequentes alterações da motilidade e de morfologia, ao nível do trato digestivo, sendo no esôfago e o cólon as manifestações mais comuns. São sinais e sintomas do esôfago: disfagia, regurgitação, epigastralgia ou dor retroesternal, odinofagia, soluço, ptialismo, emagrecimento, hipertrofia das parótidas. 0 cólon caracteriza-se por constipação intestinal, meteorismo, distensão abdominal e fecaloma. ${ }^{27,28}$ Quando o paciente associa a forma cardíaca com a digestiva, caracteriza a forma mista. 29,30,31

Também pode ocorrer a forma congênita, onde os sinais clínicos são: hepatomegalia, esplenomegalia, icterícia, equimoses e convulsões decorrentes da hipoglicemia. . $9,30,31^{2}$

\section{Tratamento}

No tratamento da infecção humana, foram testadas centenas de drogas contra a DC. ${ }^{30}$ Os primeiros compostos desenvolvidos experimentalmente para o tratamento específico da DC, após a sua descoberta em 1909, foram o atoxyl (arsênico), a tintura de fucsina, o tártaro emético (antimonial pentavalente) e o cloreto de mercúrio. Todos estes compostos se mostraram ineficazes no tratamento proposto. .11,32,33 $^{2}$

Em 1936, um derivado quinoleínico com discreta atividade parasiticida foi empregado pela primeira vez no tratamento da forma aguda da DC..$^{34,35}$

Em 1968, foi realizada uma avaliação meticulosa dos fármacos disponíveis para o T.cruzi em condições in vitro e in vivo. Com base nos resultados obtidos, 27 compostos e mais de 30 antibióticos foram considerados inativos e outros apresentaram efeito supressor da parasitemia, porém não se mostraram curativos: o bisquinaldina, as aminoquinolinas (pentaquina, isopentaquina e primaquina), os arsênicos trivalentes, os aminoglicosídeos, nitrofuranos e os antibióticos. ${ }^{36}$

No final dos anos 60 e início dos anos 70, ocorreram fatos animadores para o tratamento da $\mathrm{DC}$, surgindo às drogas nifurtimox e 0 benzonidazol. ${ }^{37} \mathrm{O}$ nifurtimox (5-nitrofurano) foi introduzido na terapêutica em $1967^{38}$ e o benzonidazol (2- nitroimidazol) em 1972.39,40

0 nifurtimox foi retirado do mercado devido aos diversos efeitos colaterais sofridos pelos pacientes, ${ }^{41}$ e desde a década de 1980, o nifurtimox teve a sua comercialização interrompida, primeiramente no Brasil e depois em outros países da América do Sul. ${ }^{33}$ Já o benzonidazol um pouco mais efetivo, apesar de apresentar moderada toxicidade, consegue eliminar os parasitas no sangue e nos tecidos, se administrados nadose certa e durante o período de dois meses na fase aguda. Porém, nenhuma droga demonstrou capacidade curativa. ${ }^{42}$

Também são citadas algumas combinações medicamentosas (tabela 1). Essas interações medicamentosas e os horários de tratamento devem ser adaptados de acordo com a observação de efeitos colaterais. ${ }^{43}$

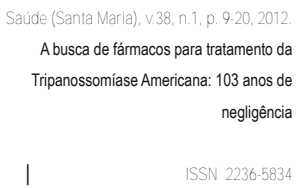


Tabela 1 - Combinações medicamentosas, utilizadas no tratamento da DC. ${ }^{43}$

\begin{tabular}{cc}
\hline Drogas e Doses & Duração \\
\hline Nifurtimox 8mg/kg/dia + Bezonidazol 5mg/kg/dia & 60 dias \\
Nifurtimox 8mg/kg/dia + Alopurinol 8-10mg/kg/dia & 60 dias \\
Bezonidazol 5mg/kg/dia + Alopurinol 8-10mg/kg/dia & 60 dias \\
Nifurtimox 8mg/kg/dia + Cetoconazol 5-6mg/kg/dia & 60 dias \\
Nifurtimox 8mg/kg/dia + Fluconazol 5-6mg/kg/dia & 60 dias \\
Nifurtimox 8mg/kg/dia + Itraconazol 5-6mg/kg/dia & 60 dias \\
Bezonidazol 5mg/kg/dia + Cetoconazol 5-6mg/kg/dia & 60 dias \\
Bezonidazol 5mg/kg/dia + Fluconazol 5-6mg/kg/dia & 60 dias \\
Bezonidazol 5mg/kg/dia + Itraconazol 5-6mg/kg/dia & 60 dias \\
\hline
\end{tabular}

Outras associações duplas ou triplas podem ser testadas, entre drogas com diferentes mecanismos de ação. Esta proposta não exclui as investigações de novas drogas para eliminação do agente etiológico, porém até a droga ideal para o tratamento específico da DC ser descoberta, necessita-se desenvolver novas estratégias para alcançar maior eficácia com as drogas antigas, usando tratamentos combinatórios e desenvolver cursos de experimentação racional para novos medicamentos. ${ }^{43}$

Os dois principais medicamentos utilizados no tratamento da DC tem mais de 40 anos desde a sua descoberta, pois muitas vezes se desestimulam investigações de terreno sobre 0 controle da transmissão e o manejo de infectados pela (DC) sob a alegação de que são poucos os investimentos, por se tratar de uma doença negligenciada e não gerar lucro para as empresas farmacêuticas. $44,45,46,47,48$

Em sua tese de doutorado, a pesquisadora Chung Man Chin, apresenta a eficiência que o composto químico nitrofural demonstra em exterminar o T.cruzi. "O nitrofural é usado como um antimicrobiano tópicd devido ao seu alto grau de toxicidade, mas apresenta alta atividade contra o parasita" diz Chung. Dessa forma, a proposta era diminuir essa toxicidade para que o composto pudesse ser utilizado por via oral e para o tratamento durante a fase crônica da doença. Para isso, Chung modificou a molécula de nitrofural, provocando uma alteração que aumentando a seletividade para o parasita. 0 nitrofural possui uma ação mutagênica elevada e baixa solubilidade, o que faz com que ele tenha de ser consumido em grandes quantidades. Esse é o motivo da toxicidade dessa substância, que pode causar hemólise (quebra das células sanguíneas), muito desconforto, doenças nervosas, entre outras alterações. "Não existem fármacos sem efeitos colaterais adversos, mas a idéia é diminuí-los ao máximo" diz Chung, que constatou que, entre os derivados sintetizados, o hidroximetilnitrofural era o mais ativo em testes realizados em células in vitro e o que apresentou menos toxicidade. ${ }^{49}$

Outros quimioterápicos foram testados como: TAK-187 e Ravuconazole, ${ }^{35,50}$ Alopurinol, ${ }^{35,51,52}$ Megazol, ${ }^{35,53}$ e Naftoimidazóis, ${ }^{35,54}$ porém nenhum teve resultados significantes sobre a sua eficiência. Já o Posaconazol, um análogo do itraconazol foi registrado na União Européia, na Australia e nos Estados Unidos como um antifúngico sistêmico sendo é considerado atualmente o mais forte candidato para novos tratamentos específicos da DC. $35,55,56$

Saúde (Santa Maria), v.38, n.1, p. 9-20, 2012 Bezerra, W. S.; Meneguetti, D. U. O.; Camargo, L. M. ISSN 2236-5843 13 
Também foram investigados os efeitos dos linfócitos B e T (CD4 e CD8) no tratamento com as drogas benzonidazol e posaconazol, mostraram que a taxa de sobrevivência de camundongos infectados com T.cruzi e tratados com os referidos compostos foi de $100 \%$, com cerca de $87 \%$ deles ficando curados após o tratamento. No entanto, os resultados apontaram que quando os camundongos infectados tinham a presença do linfócito T (CD4) em seu sistema imunológico, os parasitos reapareceram, passado o efeito da droga, e a taxa de sobrevivência caiu para $6 \%$. 0 resultado do tratamento na presença do linfócito T (CD8) foi considerado intermediário, com taxas de sobrevivência entre $81 \%$ e $86 \%$, e de cura entre $31 \%$ e $66 \%$. Já, na presença do linfócito $B$, as duas drogas tiveram efeitos distintos. $O$ tratamento com benzonidazol resultou numa taxa de $67 \%$ de sobrevivência, com $22 \%$ de cura. O posaconozol, no entanto, teve um efeito mais benéfico nesses animais, representando $71 \%$ de cura e $100 \%$ de sobrevivência. ${ }^{57}$

\section{Fitoterápicos}

Há muito tempo as plantas medicinais são utilizadas no tratamento de doenças parasitárias e, muitos trabalhos corroboram a importância terapêutica atribuída a produtos de origem vegetal, bem como descrevem a atividade tripanossomicida de vários princípios ativos naturais. ${ }^{58}$

A administração de um medicamento natural, com baixa toxicidade, ocasionaria menos efeitos colaterais no paciente. Outra vantagem seria o custo mais baixo para a população, principalmente se o medicamento fosse oriundo de um vegetal de fácil cultivo. ${ }^{58}$

Diversas classes, como quinonas, flavonóides, alcalóides e terpenos apresentam-se ativas contra o T.cruzi. Entre as quinosas podemos citar: Naftoquinona b-lapachona, presente em várias espécies vegetais da família Bignoniaceae, possuem atividade contra as formas amastigota, epimastigota e tripomastigota do parasita. $\mathrm{O}$ seu derivado de transformação química, a 3-alil-b-lapachona, é ativo contra formas tripomastigotas e tem sido sugerido como uma droga alternativa para o uso em bancos de sangue. ${ }^{59,60}$ Plumbagina, isolada de Pera benensis Rusby (Euphorbiaceae), foi $100 \%$ ativa na concentração de $250 \mu \mathrm{g} / \mathrm{mL}$ de sangue contaminado. ${ }^{60,61}$ Oxalis erythrorhiza Gillies conhecida como boldo da cordilheira, possui benzoquinona embelina e também alguns alquil fenóis. A embelina mostrou ter atividade contra o T.cruzi, com 100\% de lise dos protozoários, à uma concentração de $100 \mu \mathrm{g} / \mathrm{mL} .^{60,62}$

Dentro dos flavonóides são citados: O 3-metoxiflavonol penduletina e a flavanona sacuranetina, isolados de Trixis vauthieri, eliminaram 99 e 100\%, respectivamente, do parasita no sangue infectado, na concentração de $500 \mu \mathrm{g} / \mathrm{mL}$. ${ }^{63}$ No estrato de Lychnophora pohlii, foram isolaram sete substâncias ativas contra formas tripomastigotas de T.cruzi, dentre elas os flavonóides luteolina e vicenina-2, esses demonstraram atividade tripanossomicida significativa na concentração de $500 \mu \mathrm{g} / \mathrm{mL} .{ }^{64} \mathrm{O}$ extrato de Lychnophora staavioides forneceu 10 flavonóides: que foram testados contra o T.cruzi, sendo que a substância mais ativa foi a quercetina-3-metil éter, que não causou lise de células sanguíneas e na dose de $500 \mu \mathrm{g} / \mathrm{mL}$ mostrou-se promissora para uso contra T.cruzi em bancos de sangue. ${ }^{65}$

Alguns alcalóides com atividade tripanossomicida também são citados como: Os 2-n-

Saúde (Santa Maria), v.38, n.1, p. 9-20, 2012 A busca de fármacos para tratamento da Tripanossomiase Americana: 103 anos de negligência propilquinolina, chimanina B e chimanina D, isolados de Galipea longiflora, apresentaram atividades similares às das drogas de referência nifurtimox e benznidazol, contra cinco cepas 
de formas epimastigotas do T.cruzi.60,66 Os cocsolina, dafnandrina e dafnolina, isolados de Albertisia papuana e girocarpina e feantina, isolados de Gyrocarpus americanus demonstraram boa atividade anti T.cruzi. ${ }^{61}$

Vários terpenos são relatados em estudos de compostos tripanossomicida tais como: 0 ácido caurenóico isolado de Mikania obtusata, ${ }^{67}$ Xylopia frutescens e de Viguiera aspillioides ${ }^{68}$ apresentou $100 \%$ de atividade contra o T.cruzi, na concentração de $1000 \mu \mathrm{g} / \mathrm{mL}$ de sangue contaminado. ${ }^{67,68}$ Outros terpenos com ação anti T.cruzi são: 0 ácido xilópico, isolado de Xylopia frutescens, caurenol, isolado de Viguiera aspillioides e de Xylopia frutescens, ${ }^{68}$ os diterpenos 17-hidroxicauranol e o ácido traquilobânico, isolados de Viguiera aspillioides, terpenóides isolados de Mikania stipulacea e Mikania hoehnei, ${ }^{69}$ os diterpenos komarovispirona, dracocequinonas isolados de Dracocephalum komarovi, ${ }^{70}$ o taxol, um diterpeno isolado de Dracocephalum komarovi e o triterpeno tingenona, também extraído da mesma espécie. ${ }^{59}$

Uma substância usada em cosméticos - a carragenina, derivada de algas do mar irlandesas tem sido considerada um potencial ativo que poderá contribuir para 0 desenvolvimento de novos fármacos contra a (DC). Extratos brutos de espécies vegetais brasileiras, especialmente da família Lauraceae, já mostraram, em ensaios in vitro, que podem ser úteis no combate ao T.cruzi, cujo crescimento chegou a ser inibido em até $100 \%$. Substâncias sintéticas, como as diamidinas aromáticas, também têm sido testadas contra 0 parasito, demonstrando alta atividade e seletividade. ${ }^{71,72}$

Em estudo recente pesquisadores testaram in vitro o efeito dos extratos brutos de 92 espécies vegetais brasileiras contra o parasito T.cruzi, causador da DC. Dos extratos testados, 11 apresentaram resultados satisfatórios, sendo que sete inibiram o crescimento do parasito entre $50 \%$ e $90 \%$ e quatro atingiram uma inibição de $100 \% .58$

Diversas plantas são citadas por apresentarem ação anti-T.cruzi, entre elas podemos citar: Camellia sinensis, ${ }^{73}$ Baccharis trimera, Cymbopogon citratus, Matricaria chamomilla, Mikania glomerata, Ocimum gratissimum, Piper regnellii, Prunus domestica, Psidium guajava, Sambucus canadensis, Stryphnodendron adstringens, Tanacetum parthenium, e Tanacetum vulgare ambas apresentam efeito significativo contra 0 parasita, com a porcentagem de inibição de crescimento entre 49,5 e $99 \%$. Os extratos não mostraram efeito citotóxico em hemácias de carneiro. Essas plantas medicinais podem ser fontes alternativas de novos compostos clinicamente ativos contra T.cruzi. ${ }^{74}$

Na região amazônica podemos citar também a Banisteriopsis caapi e Psychotria viridis que tem ação anti-tripanosomal contra o Trypanosoma lewisii ${ }^{75,76}$ e T.cruzi. $^{76,77}$ Essas plantas são utilizadas no preparo de um chá conhecido como (Ayahuasca) que é utilizado em rituais religiosos na região amazônica, tanto por indígenas como pela população local, ${ }^{76} \mathrm{e}$, acredita se que é devido ao uso desse chá que indígenas não se infectam por T.cruzi e Leishmania sp.

\section{Considerações Finais}

Já se passaram mais de 100 anos da descoberta da DC, e até hoje não foi desenvolvido nenhum fármaco com potencial satisfatório de cura, sendo que o medicamento ainda utilizado é o benzonidazol, porém o mesmo apresenta alguns efeitos colaterais.

Saúde (Santa Maria), v.38, n.1, p. 9-20, 2012. Bezerra, W. S.; Meneguetti, D. U. O.; Camargo, L. M. A. ISSN 2236-5843 15 
Devido o pouco investimento na produção de novos fármacos para o tratamento da (DC), pesquisadores direcionam seus esforços para combinações entre os medicamentos já conhecidos entre eles, como o Nifurtimox, para tentar obter melhores resultados ${ }^{43}$, porém nenhuma dessas combinações alcançou índices elevados de cura em todos os estágios de evolução da doença.

Entre os quimioterápicos o Posaconazol, é o mais forte candidato para novos tratamentos específicos da DC. ${ }^{35,55,56} \mathrm{Em}$ testes com camundongos obteve-se $71 \%$ de cura e $100 \%$ de sobrevivência, e no mesmo estudo com o benzonidazol, medicamento de referência, o resultado foi de apenas $67 \%$ de sobrevivência, com $22 \%$ de cura. ${ }^{57}$

Um dos métodos alternativos utilizados pela população para o tratamento e prevenção da DC é o uso de fitoterápicos, entre eles podemos citar: Banisteriopsis caapi e Psychotria viridis, que são típicos da região amazônica, no entanto, o uso desses vegetais preocupa, pois na maioria das espécies não foram feitos testes citotoxicidade e mutagenicidade.

É importante destacar a farmacobotânica, farmacognosia, genética e toxicologia, como áreas potenciais para realização de pesquisas com vegetais, pois nos mesmos, são encontrados princípios ativos, que podem ter potencial de cura para várias doenças, em especial a DC. E, se tratando da região amazônica onde apenas $5 \%$ dos seus vegetais foram estudados, a área de pesquisa é promissora, principalmente para a produção de fármacos no tratamento das doenças negligenciadas, que é uma realidade da região.

\section{Referências}

1. Costa M, Dias J.C.P. A descoberta da doença de chagas. Cad de Ciên e Tecn, v. 16(2), p. 11-51, 1999.

2. Jurberg J, Galvão C, Noireal F, Carvalho R, Rocha D, Lent $H$. Uma Iconografia dos Triatomíneos (Hemíptera: Reduviidae). Entomol Vectores, v. 11(3), p. 454-494, 2004.

3. Tartarotti E, Tercília M, Vilela AO, Ceron CR. Problemática vetorial da Doença de Chagas. Arq Ciênc Saúde, v. 11(1), p. 44-7, 2004.

4. Fiocruz. Centenário Fundação Osvaldo Cruz. Ministerio da Saúde, 2000.

5. Neto VA, Pasternak J. Centenário da doença de Chagas. Rev Saú Púb, v. 43(2), p. 381-382, 2009.

6. MSF. Informativo médicos sem fronteiras. v. 24, 2009.

7. Fiocruz. Agência Fiocruz de Notícia. OMS inaugura iniciativa inédita para controle global da doença de chagas [online]. 2008. [acesso 2010 Mar 27]. Disponível em http://www.fiocruz.br/ccs/cgi/cgilua.exe/sys/start.htm

8. Guhl F., Vallejo GA. Interruption of Chagas disease transmisión in the Andean Countries: Colombia. Mem Inst Oswaldo Cruz, v. 94, p. 413, 1999.

9. Morel CM, Lazdins J, Chagas D. Nature Reviews Microbiology. London: Nature Publishing Group, p. 14-15, 2003.

10. Dndi - Drugs for Neglected Diseases Initiative [online]. 2003. [acesso 2006 out 5]. Disponível em: $<$ http://www.dndi.org.br/>

Saúde (Santa Maria), v.38, n.1, p. 9-20, 2012. A busca de fármacos para tratamento da Tripanossomiase Americana: 103 anos de negligência
11. Sobrinho JLS, Medeiros FPM, La Roca MF, Silva KER, Lima LNA, Neto PJR. Delineamento de alternativas terapêuticas para o tratamento da doença de Chagas. Rev Pato trop, v. 36 (2), p.103-118, 2007. 
12. Argolo AM, Felix M, Pacheco R, Costa J. Doença de chagas e seus principais vetores no Brasil. Fundação Oswaldo Cruz. Programa Integrado de Doença de Chagas (PIDC). Instituto Oswaldo Cruz. Ação comemorativa do centenário de descoberta da doença de Chagas. Fundação Carlos Chagas Filho de Amparo à Pesquisa do Estado do Rio de Janeiro. Rio de Janeiro, 2008.

13. Guhl F, Schofield CJ. Population genetics and control of Triatominae. Parasitol Today, v. 12, p. 169-70, 1996.

14. Oxford. World Development Report . Investing in Health. Oxford University Press, New York. p.329, 1993.

15. Dias JCP, Silveira AC, Schofield CJ. The Impact of Chagas Disease Control in Latin America. Mem Inst Oswaldo Cruz, v. 97(5), p. 603-612, 2002.

16. Dias E, Dias JCP, Garcia ALR, Dias RB, Machado ENM, Gouveia SC. Doença de Chagas: Textos de apoio. Brasília: Ministério da Saúde. Superintendência de Campanhas de Saúde Pública, 1989.

17. Dias JCP, Diotaiuti L. Triatomíneos e seu controle no Brasil: perspectivas e desafios. Cad Saú Pub, v. 16, p. 1-126, 2000.

18. Reiche EMV, Inouye MMZ, Pontello R, Morimoto HK, Itow JS, Matsuo T et al. Seropositivity for antiTrypanosoma cruzi antibodies among blood donors of the. Hospital Universitário Regional do Norte do Paraná, Londrina, Brazil . Inst Med Trop, v. 38, p. 233-240, 1996.

19. Colli W, Alves MJM. Chagas um repensar de abordagens experimentais. Médicus HC-FMUSP, v. 1, p. 75-7, 1998.

20. Storino R, Milei J. Evolución natural y estudios longitudinales. In: Storino R, Milei J, Doyma (eds) Enfermedad de Chagas. Argentina. p.593-604, 1994.

21. Suasnábar DF, Arias E, Streiger M, Barco M, Amicone N, Miglietta H. Evaluación de la quimioterapia especifica en infectados chagásicos adultos en fase indeterminada con más de quince años de seguimiento. Rev de la Fed Arg de Cardio, v. 30, p. 496-503, 2001.

22. Streiger ML, Barco ML, Fabbro DL, Arias ED, Amicone NA. Estudo longitudinal e quimioterapia específica em crianças, com doença de Chagas crônica, residentes em área de baixa endemicidade da República Argentina. Rev. Soc. Bras Med Trop, v. 37(5), p. 365-375, 2004.

23. Chagas CRJ. Tripanosomíase Americana: Forma aguda da moléstia. Mem Inst Oswaldo Cruz, v. 8, p. 37-69, 1916.

24. Pereira VL, Marcos DAA, Boainain E. Xenodiagnostico, Hemocultura e teste de lise mediada pelo complemento, como critérios de seleção de pacientes chagásicos crônicos para quimioterapia. Rev Inst Med Trop São Paulo, v. 31, p. 301-307, 1989.

25. Mirtha LS, Barco ML, Fabbro DL, Arias ED, Amicone NA. Estudo longitudinal e quimioterapia específica em crianças, com doença de Chagas crônica, residentes em área de baixa endemicidade da República Argentina. Rev Soc Bras Med Trop, v. 37(5), p. 365-375, 2004.

26. Macedo VO. Forma indeterminada da doença de Chagas. In: Dias JCP, Coura JR (eds). Clínica e terapêutica da doença de Chagas. Rio de Janeiro, p. 135-151, 1997.

27. Brasil. Guia de vigilância epidemiológica. Serie -A. Normas e manuais técnicos. Secretaria de Vigilância em Saúde. Ministério da Saúde. Brasília, 2005.

28. Brasil. Atenção Básica. Vigilância em saúde: zoonoses. Ministério da Saúde, Secretaria de Atenção à Saúde, Departamento de Atenção Básica. Brasília, 2009. 15p.

29. Dias E. Os efeitos da superinfecção sobre a evolução da cardiopatia crônica chagásica. Rev Goiana Med,supl. 9, p. 233-239, 1962.

30. Coura JR. Tripanosomose, Doença de Chagas. Cienc Cult, v. 55(1), p. 30-33, 2003.

Saúde (Santa Maria), v.38, n.1, p. 9-20, 2012. Bezerra, W. S.; Meneguetti, D. U. O.; Camargo, L. M. A.

31. Massaro DC, Rezende DS, Camargo LMA. Estudo da Fauna de Triatomíneos e da Ocorrência de $155 \mathrm{~N}$ 2236-5843 Doença de Chagas em Monte Negro, Rondônia, Brasil. Rev Bras Epidemiol, v. 11, p. 228-240, 2008. 
32. Croft SL. Pharmacological Approaches to Antitrypanosomal Chemotherapy. Mem Inst Oswaldo Cruz, v. 94, p. 215-220, 1999.

33. Coura JR, Castro SL. A Critical Review on Chagas Disease Chemotherapy. Mem Inst Oswaldo Cruz, v. 97, p. 3-24, 2002.

34. Mazza S, Cássio R, Zucardi EL. Primer caso agudo de enfermedad de Chagas comprobado em Tucumán y su tratamiento com Bayer 7602. Misión de Estudios de Patologia Regional (MEPRA) v. 32, p. 3-18, 1937.

35. Oliveira M.F, Dias ATN, Pontes VMO. Júnior, A.S.S., Coelho, H.L.L., Coelho, I.C.B. Tratamento etiológico da doença de chagas no Brasil. Rev Pato Trop, v. 37 (3), p. 209-228, 2008.

36. Brener Z. Terapêutica experimental da doença de Chagas. In: CANÇADO, J.R. Doença de Chagas. Belo Horizonte. p. 510-16, 1968.

37. Dias JCP, Schofield CJ. The evolution of Chagas disease (American trypanosomiasis) control after 90 years since Carlos Chagas discovery. Mem Inst Oswaldo Cruz, v. 94 suppl. I, p. 103-121, 1999.

38. Bock M, Gonert R, Haberkorn A. Studies with Bay 2502 on animals. Bol Chil Parasitol, v. 24, p. 13-19, 1969 .

39. Schofield CJ, Jannin J, Salvatella R. The future of chagas disease control. Trends Parasitol, v. 22, p. 583$588,2006$.

40. Croft SL, Barret MP, Urbina JA. Chemotherapy of trypanosomiases and leishmaniasis. Trends Parasitol, v. 21, p. 508-512, 2005.

41. Rassi A, Amato-Neto V, Siqueira AF, Ferrioli-Filho F, Amato VS, Rassi GG, Rassi JA. Tratamento da fase crônica da doença de Chagas com nifurtimox associado a corticóide. Rev Soc Bras Med Trop, v. 35, p. 547$550,2002$.

42. Rassi A, Amato NVA, Siqueira AF, Ferriolli FF, Amato VS, Rassi JA. Efeito protetor do benznidazol contra a reativação parasitária em pacientes cronicamente infectados pelo Trypanosoma cruzi e tratados com corticóide em virtude de afecções associadas. Rev Soc Bras Med Trop, v. 32, p. 475-482, 1999.

43. Coura JR. Present situation and new strategies for Chagas disease chemotherapy - a proposal. Mem Inst Oswaldo Cruz, v. 104(4), p. 549-554, 2009.

44. Dias JCP. Doença de Chagas e a questão da tecnologia. Bol Oficina Sanit Panam, v. 99, p. 244-257, 1985.

45. Vieira C. Globalización, comercio internacional y equidad en materia de salud. Rev Panam Salud Pública, v. 11, p. 425-429, 2002.

46. Jörg M, Storino RA. La enfermedad de Chagas em el siglo XXI: consenso para una asignatura pendiente. Consenso Argentino sobre la Enfermedad de Chagas. Rev Argent Cardiol 2002; 70:9-10.

47. Dias JCP. Globalização, iniquidade e doença de Chagas. Cad Saude Publica, v. 23, p.13-22, 2007.

48. Malafaia G, Rodrigues ASL. Centenário do descobrimento da doença de Chagas: desafios e perspectivas. Rev Soc Bras Med Trop, v. 43(5), p. 483-485, 2010.

49. Bosquesi PL, Almeida AE, Blau L, Menegon RF, Santos JL, Chung MC. Toxicidade de fármacos nitrofurânicos. Rev Ciênc Farm Básica Apl, v. 29(3), p. 231-238, 2008.

50. Urbina JA, Docampo R. Specific chemotherapy of Chagas disease: controversies and advances. Trends Parasitol, v. 19, p. 495-501, 2003.

51. Gianella A, Holzman A, Liosh N, Barja ZY, Peredo C. Eficácia del alopurinol en la enfermedad de Chagas crônica. Resultados del estudio realizado en Santa Cruz, Bolivia. Bol Cientif Cenetrop, v. 16, p. 25-30, 1997.

(Santa Maria), v.38, n.1, p. 9-20, 2012 A busca de fármacos para tratamento da Tripanossomiase Americana: 103 anos de negligência ISSN 2236-5834
52. Rassi A, Luquetti AO, Rassi AJR, Rassi GG, Rassi SG, Da Silva IG, Rassi AG. Specific treatment for Trypanosoma cruzi: lack of efficacy of allopurinol in the human chronic phase of Chagas disease. Am J Trop Med Hyg, v. 76, p. 58-61, 2007 
53. De Castro SL, Meirelles MN. Mechanism of action of a nitroimidazole-thiadiazole derivate upon Trypanosoma cruzi tissue culture amastigotes. Mem Inst Oswaldo Cruz, v. 85, p. 95-99, 1990.

54. Furtado F. Dobradinha contra Chagas. Ciência Hoje, v. 39, p. 44-45, 2006.

55. Urbina JA, Payares G, Contreras LM, Liendo A, Sanoja C, Molina J et al. Antiproliferative effects and mechanism of action of SCH 56592 against Trypanosoma (Schizotrypanum) cruzi: in vitro and in vivo studies. Antimicrob Agents Chemother, v. 42, p. 1771-1777, 1998.

56. Molina J, Filho OM, Brener Z, Romanha AJ, Loebenberg D, Urbina JA. Activities of the triazole derivate $\mathrm{SCH} 56592$ (Posaconazole) against drug-resistant strains of the protozoan parasite Trypanosoma (Schizotrypanum) cruzi in immunocompetent and immunosuppressed murine host. Antim Agents Chemoth v. 44 , p. $150-155,2000$.

57. Ferraz ML, Gazzinelli RT, Alves RO, Urbina JA, Romanha A . Absence of CD4+ T lymphocytes, C8+ T lymphocytes, or B lymphocytes has different effects on the efficacy of Posaconazole an Benznidazole in treatment of experimental acute Trypanosoma cruzi infection. Antimicrobial Agents and Chemotherapy, v. 53, p. 174-179, 2009

58. II, Marques F. Plantas brasileiras se mostram promissoras contra o parasito causador da doença de Chagas. Agencia Fiocruz de Noticias [online]. 2009. [acesso 2010 Mar 27] Disponível em $<$ http://www.fiocruz.br/ccs/cgi/cgilua.exe/sys/start.htm?infoid=2641\&sid=9>

59. Castro SL. The challenge of Chagas'disease chemotherapy: An update od drugs assayed against Trypanosoma cruzi. Acta Tropica, v. 53, p. 83-98, 1993.

60. Guimarães DAS, Faria AR. Substâncias da natureza com atividade anti-Trypanosoma cruzi. Rev Bras Farmacogn, v. 17(3), p. 455-465, 2007.

61. Fournet A, Rojas AA, Inchausti A, Ascurrat M, Fleitas N, Rodriguez E. In vitro activity and mutagenicity of bisbenzilisoquinolines and quinones against Trypanosoma cruzi Trypomastigotes. Phytother Res, v. 8, p. 141144, 1994.

62. Feresin GE, Tapia A, Sortino M, Zacchino S, Arias AR, Inchausti A, Yaluff G, Rodriguez J, Theoduloz, C., Schmeda-Hirschmann G. Bioactive alkyl phenols and embelin from Oxalis erythrorhiza. J Ethnopharmacol, v. 88, p. 241-247, 2003.

63. Ribeiro A, Santos LMST, Romanha AJ, Veloso DP, Zani CL. Trypanocidal flavonoids from Trixis vauthieri. J Nat Prod, v. 60, p. 836-838, 1997.

64. Grael CFF, Albuquerque S, Lopes JL. Chemical constituents of Lychnophora pohlii and trypanocidal activity of crude plant extracts and of isolated compounds. Fitoterapia, v. 76, p. 73-82, 2005.

65. Takeara R, Albuquerque S, Lopes NP, Lopes JLC. Trypanocidal activity of Lychnophora staavioides Mart.(Vernonieae, Asteraceae). Phytomedicine, v. 10, p. 490-493, 2003.

66. Fairlamb AH. Trypanotione metabolism in Trypanosoma cruzi. Mem Inst Oswaldo Cruz, v. 89 (Suppl. I), p. 37,1994

67. Alves TMA, Chaves PPG, Santos LMST, Nagem TJ, Murta SMF, Ceravolo IP, Romanha AJ, Zani CL. A diterpene from Mikania obtusata active on Trypanosoma cruzi. Planta Med, v. 61, p. 85-87, 1995.

68. Takahashi JA, Boaventura MAD, Oliveira AB, Chiari E, Vieira HS. Isolamento e atividade tripanossomicida de diterpenos caurânicos de Xylopia frutescens Aubl. 17 Reunião da Sociedade Brasileira de Química, 1994.

69. Nascimento AM, Chaves JS, Albuquerque S, Oliveira DCR. Trypanocidal properties of Mikania stipulacea and Mikania hoehnei isolated terpenoids. Fitoterapia, v. 75, p. 381-384, 2004.

70. Uchiyama N, Ito M, Kiuchi F, Honda G, Takeda Y, Khodzhimatov OK et al. A trypanocidal diterpene with novel skeleton from Dracocephalum komarovi. Tetrahedron Lett, v. 45, p. 531-533, 2004.

Saúde (Santa Maria), v.38, n.1, p. 9-20, 2012 Bezerra, W. S.; Meneguetti, D. U. O.; Camargo, L. M.

71. Marques F. Novos tratamentos e terapias contra doença de Chagas serão apresentados em simpósio.

Agencia Fiocruz de Noticias [online]. 2009. [acesso em 23 de Mar 2011]. Disponível em $<$ <ttp://www.fiocruz.br/ccs/cgi/cgilua.exe/sys/start.htm?infoid=2639\&sid=9\&tpl=printerview> 
72. Valverde R. Substância usada em cosméticos poderá contribuir para o desenvolvimento de novos fármacos para a doença de Chagas. Agencia Fiocruz de Noticias [online]. 2009. [acesso 2011 Nov 3]. Disponível em <http://www.fiocruz.br/ccs/cgi/cgilua.exe/sys/start.htm?infoid=2644\&sid=9 >

73. Paveto C, Guida MC, Esteva MI. Martino V, Coussio J, Flawiá MM, Torres HN. Anti-Trypanosoma cruzi Activity of Green Tea (Camellia sinensis) Catechins. Antimicrob Agents Chemother, v.48(1), p. 69-74, 2004.

74. Luize PS, Tiuman TS, Morello LG, Maza PK, Nakamura TU, Filho BPD et al. Effects of medicinal plant extracts on growth of Leishmania (L.) amazonensis and Trypanosoma cruzi. Rev Bras Ciên Farm, v. 41 (1), p. 85-94, 2005.

75. Mckenna DJ, Callaway JC, Grob CS The scientific investigation of Ayahuasca: a review of past and current research. The Heffer Review of Psychedelic Research, v. 1, p. 65-77, 1998.

76. Sérpico RL, Camurça DM, Zanzini ES. Ayahuasca: revisão teórica e considerações botânicas sobre as espécies. Monografia, conclusão do curso de Licenciatura Plena em Ciências Biológicas pela Universidade Guarulhos, 2006.

77. Pomilio A B, Vitale AA, Ciprian-Ollivier J, Cetkovich-Bakmas M, Gómez R, Vázquez G. Ayahoasca: an experimental psychosis that mirrors the transmethylation hypothesis of schizophrenia. Journal of Ethnopharmacology, v. 65, p. 29-51, 1999.

\section{Whatani Silva Bezerra}

Endereço para correspondência - Rua: Rio Grande do Sul, № 4098, Setor: 5, Ariquemes - Rondônia, CEP: $76170-970$

E-mail: whatani_sb@hotmail.com

Currículo Lattes: http://lattes.cnpq.br/5492693622857279

Recebido em 13 de março de 2012.

Aprovado em 06 de junho de 2012. 